\title{
Atmosfera modificada e refrigeração para conservação pós-colheita da nêspera 'Fukuhara'
}

\author{
Juliana Sanches ( $\left.{ }^{1,{ }^{*}}\right)$; Patrícia Cia (1); Silvia Regina de Toledo Valentini (2); Eliane Benato (2); \\ Edvan Alves Chagas ( $\left.{ }^{3}\right)$; Rafael Pio $\left({ }^{4}\right)$ \\ (') Instituto Agronômico (IAC), Centro de Engenharia e Automação, Caixa Postal 26, 13201-970 Jundiaí (SP). \\ (2) Instituto de Tecnologia de Alimentos (ITAL), Caixa Postal 139, 13070-178 Campinas (SP). \\ (3) Empresa Brasileira de Pesquisa Agropecuária, Centro de Pesquisa Agroflorestal de Roraima (CPAF), 69301-970 Boa Vista (RR). \\ (4) Universidade Federal de Lavras (UFLA), Departamento de Agricultura, Caixa Postal 3037, 37200-000 Lavras (MG). \\ (*) Autora correspondente: jsanches@iac.sp.gov.br
}

Recebido: 16/abr./2009; Aceito: 25/set./2010.

\begin{abstract}
Resumo
A rápida perda de qualidade pós-colheita limita a comercialização da nêspera no mercado de frutas frescas. Neste sentido, este trabalho teve por objetivo avaliar os efeitos da utilização de atmosfera modificada na conservação pós-colheita da nêspera 'Fukuhara' armazenada sob refrigeração ( $1{ }^{\circ} \mathrm{C} / 90 \%$ UR). Para tanto, os frutos ( $\left.\approx 450 \mathrm{~g}\right)$ foram acondicionados em bandejas de poliestireno e embalados em dois tipos de filme: polietileno de baixa densidade (PEBD, 50 um) e polipropileno (PP, $30 \mu \mathrm{m}$ ), sendo, em seguida, armazenados a $1{ }^{\circ} \mathrm{C} / 90 \%$ UR. Os frutos foram comparados a um controle que não envolveu a aplicação de filmes. As análises físico-químicas (perda de massa, firmeza, sólidos solúveis, pH e acidez titulável) foram realizadas aos 15, 30 e 45 dias sob refrigeração, seguido por período de três dias sob condição ambiente $\left(25^{\circ} \mathrm{C} / 80 \%\right.$ UR). A composição gasosa $\left(\mathrm{O}_{2} / \mathrm{CO}_{2}\right)$ no espaço livre das embalagens, a incidência de podridões e de manchas escuras na epiderme dos frutos também foram avaliadas. Os resultados mostraram que a utilização de filme PEBD e PP para a conservação pós-colheita da nêspera, apesar de ser eficaz na redução da perda de massa e não alterar a cidez titulável, o pH e a firmeza dos frutos, não é indicada para o aumento do período de conservação, pois deprecia sua qualidade devido a condições de anaerobiose e elevada incidência de podridões.
\end{abstract}

Palavras-chave: Eriobotrya japonica, embalagem, armazenamento.

\section{Modified atmosphere and refrigeration for the postharvest conservation of 'Fukuhara' loquat}

\footnotetext{
Abstract

Loquats have a short period of commercialization because of the quick loss of quality after harvest. The goal of this work was to evaluate the use of modified atmosphere on postharvest conservation of 'Fukuhara' loquat stored under refrigeration $\left(1{ }^{\circ} \mathrm{C} / 90 \% \mathrm{RH}\right)$. Fruits ( $\left.\approx 450 \mathrm{~g}\right)$ were packed in polystyrene trays covered with low density polyethylene (50 $\mu \mathrm{m}$-LDPE) or polypropylene (PP, $30 \mu \mathrm{m})$ and stored under refrigeration $\left(1{ }^{\circ} \mathrm{C} / 90 \% \mathrm{RH}\right)$. Fruits were compared to a control that was not involved with film. Physico-chemical parameters (weight loss, firmness, total soluble solids, $\mathrm{pH}$, and titratable acidity) were carried out after 15,30 , and 45 -days under refrigeration, followed by three more days at room condition $\left(25^{\circ} \mathrm{C} / 80 \% \mathrm{RH}\right)$. Headspace gas composition $\left(\mathrm{O}_{2} / \mathrm{CO}_{2}\right)$, incidence of decay and spots on epidermis were also evaluated. The results showed that LDPE and PP films were effective in reducing weight loss and did not cause changes in titratable acidity, pH and firmness. However, these films were not effective for the postharvest conservation of loquat due to the development of anaerobic conditions and the increase of the disease incidence, being both harmful for the fruit quality.
}

Key words: Eriobotrya japonica, packaging, storage. 


\section{INTRODUÇÃO}

A nespereira (Eriobotrya japonica Lindl.) origina-se da Ásia (Japão, China e Índia), onde é intensamente cultivada (LiN et al., 1999). As maiores regióes produtoras mundiais estâo localizadas nos países orientais. No continente americano, o Brasil é produtor de destaque de nêsperas e o Estado de São Paulo é o líder na produção nacional, com cerca de 3,7 milhóes de caixas de $5 \mathrm{~kg}$ produzidas por ano (Pio et al., 2007). A nespereira é encontrada em 27 municípios do Estado de São Paulo, constituindo-se na oitava frutífera de clima temperado-subtropical mais cultivada em terras paulistas. Suas principais cultivares são: Mizuho, Precoce de Itaquera (Fukuhara) e Precoce de Campinas (IAC 16531) (Barbosa et al., 2003). A nêspera 'Fukuhara' é a mais cultivada no Estado de São Paulo (Pıo et al., 2008).

O aumento do consumo de nêsperas está relacionado principalmente ao sabor peculiar dos seus frutos, bem como as suas características funcionais. As nêsperas são ricas em flavonóides, ácido galacturônico, málico e fumárico, carotenóides e outros e compostos antioxidantes, localizados na casca e na polpa dos frutos (FARIA et al., 2009; Ferreres et al.,2009). Quando a nespereira recebe manejo adequado com ensacamento e desbaste dos frutos, alcança excelente qualidade e aceitação para o comércio 'in natura' (BRACKMANN et al., 1996).

$\mathrm{O}$ armazenamento refrigerado permite conservar os frutos da nespereira por períodos não muito longos, devido à sua alta perecibilidade. A refrigeraçấo pode prolongar o período de conservação das nêsperas, mas não inibe completamente a perda de massa e o declínio nos níveis de ácidos orgânicos durante armazenamento (DiNG et al., 1998). Segundo estes mesmos autores, as nêsperas cv. Mogi devem ser armazenadas por até 30 dias a baixas temperaturas $\left(1{ }^{\circ} \mathrm{C}\right.$ e $\left.5{ }^{\circ} \mathrm{C}\right)$ para minimizar a perda de massa e reduzir as mudanças composicionais associadas à qualidade da fruta. Segundo CaI et al. (2006a), frutos da nespereira são sensíveis às desordens fisiológicas promovidas pelas baixas temperaturas (chilling), fator limitante ao armazenamento por longos períodos. As injúrias pelo frio são manifestadas pela adesáo da casca à polpa, perda de suco e escurecimento interno. Como alternativa, tem-se a modificaçáo da atmosfera, cujo objetivo é estender o período de conservação dos frutos através da redução da taxa respiratória. A diminuição da taxa respiratória e consequente redução das reaçôes metabólicas, promovidas pela diminuição nos níveis de $\mathrm{O}_{2} \mathrm{e}$ aumento de $\mathrm{CO}_{2}$, atrasam o amadurecimento e a senescência dos frutos por reduzirem a utilizaçáo de carboidratos, ácidos orgânicos e outras reservas (Матнооко, 1996). A atmosfera modificada pode ainda suprimir o desenvolvimento de podridōes por atuar direta e/ou indiretamente sobre os patógenos (Cia e Benato, 2006).

BrackMANn et al. (1996) constataram que o armazenamento de nêsperas 'Mizuho' sob atmosfera controlada, a $2{ }^{\circ} \mathrm{C}$ proporcionou melhor manutenção da acidez e me- nor perda de massa, porém estimulou maior ocorrência de podridôes e manchas com depressão na epiderme. Melo e Lima (2003) verificaram que o armazenamento de nêsperas em PVC $(20 \mu \mathrm{m})$ a $3{ }^{\circ} \mathrm{C}$ foi adequado para a conservação dos frutos por um período de até 50 dias.

O objetivo deste trabalho foi testar a hipótese de que a atmosfera modificada e a refrigeração podem atuar positivamente na conservação pós-colheita de nêspera devido à redução da respiração, atrasando o processo de senescência e, consequentemente, dificultando o desenvolvimento de patógenos. Assim, avaliaram-se os efeitos de composiçóes gasosas, obtidas através da utilização de dois filmes plásticos, aliado ao armazenamento refrigerado na conservação pós-colheita de nêspera 'Fukuhara'.

\section{MATERIAL E MÉTODOS}

Frutos da nespereira 'Fukuhara', provenientes de Mogi das Cruzes (SP), foram transportados ao laboratório, onde foram selecionados quanto à ausência de defeitos, podridóes e uniformidade de cor. Os frutos, em número de nove por repetição $(\approx 450 \mathrm{~g})$, foram acondicionados em bandejas de poliestireno e embalados em dois tipos de filme: polietileno de baixa densidade (PEBD, $50 \mu \mathrm{m})$ e polipropileno (PP, $30 \mu \mathrm{m}$ ), sendo, em seguida, armazenados sob refrigeração $\left(1{ }^{\circ} \mathrm{C} \pm 1{ }^{\circ} \mathrm{C} / 90 \% \pm 5 \%\right.$ UR). Os frutos testemunha foram mantidos sob as mesmas condiçóes, em bandejas de poliestireno, mas não envoltos em filmes. As análises físico-químicas (perda de massa, firmeza, sólidos solúveis, $\mathrm{pH}$ e acidez titulável) foram realizadas aos 15,30 e 45 dias sob refrigeração, seguido por período de três dias sob condiçôes ambiente $\left(25^{\circ} \mathrm{C} \pm 2{ }^{\circ} \mathrm{C} / 80 \% \pm\right.$ $5 \%$ UR). A perda de massa (\%) foi obtida pela diferença entre massa final e inicial, determinada em balança semianalítica Mettler Toledo-PB 3002; a firmeza de polpa (N) foi determinada em texturômetro TA-XT2, ponteira $3 \mathrm{~mm}$, com distância e velocidade de penetração de $5 \mathrm{~mm}$ e $1 \mathrm{~mm} / \mathrm{s}$ respectivamente, efetuando-se a leitura em dois pontos na região equatorial dos frutos, após a retirada da casca; o teor de sólidos solúveis (\%) foi obtido em refratômetro manual Atago; o $\mathrm{pH}$ foi determinado em $\mathrm{pHmetro}$ Micronal B-274 e a acidez titulável (\% ácido málico) por titulação com $\mathrm{NaOH}(0,5 \mathrm{~N})$ até $\mathrm{pH} 8,1$.

Além destas análises, a composição gasosa $\left(\mathrm{O}_{2} / \mathrm{CO}_{2}\right)$ no espaço-livre das embalagens foi determinada com auxílio de um analisador de gases PBI Dansensor, utilizando-se alíquotas de $30 \mathrm{~mL}$ de gás, obtidas do interior das embalagens, através de coleta via septo. Após a análise da composição gasosa, realizadas a cada 15 dias sob armazenamento refrigerado, os frutos foram utilizados para as análises físico-químicas. A incidência de podridōes (porcentagem de frutos com sintomas de podridão) e de manchas escuras (porcentagem de frutos com presença de mancha) na epiderme dos frutos também foi avaliada. 
O delineamento experimental foi inteiramente casualizado com quatro repetiçóes compostas por nove frutos como unidade experimental. As médias dos dados obtidos foram submetidas à análise de variância e comparados pelo teste de Tukey a $5 \%$ de probabilidade.

\section{RESULTADOS E DISCUSSÃO}

$\mathrm{O}$ acondicionamento de nêsperas em PEBD $50 \mu \mathrm{m}$ e PP $30 \mu \mathrm{m}$ causou redução nos níveis de $\mathrm{O}_{2}$ (Figura 1a) e rápido acúmulo de $\mathrm{CO}_{2}$ (Figura 1b). Em armazenamento refrigerado, os níveis médios de $\mathrm{O}_{2}$ atingidos após o estabelecimento do equilíbrio da atmosfera foram $2,87 \% \mathrm{e}$ $0,49 \%$, para PEBD $50 \mu \mathrm{m}$ e PP $30 \mu \mathrm{m}$ respectivamente, enquanto os níveis médios de $\mathrm{CO}_{2,}, 5,08 \%$ e $21,22 \%$. A transferência dos frutos para condição ambiente $\left(25^{\circ} \mathrm{C}\right)$ causou aumento imediato nos níveis de $\mathrm{CO}_{2}$ para $10,14 \%$ (PEBD $50 \mu \mathrm{m}$ ) e $27,45 \%$ (PP $30 \mu \mathrm{m}$ ). Constatou-se redução nos níveis de $\mathrm{O}_{2}$ para $1,71 \%$ para o PEBD e um pequeno aumento para o PP $(1,16 \%)$, após transferência das frutas para condição ambiente. A baixa concentração de $\mathrm{O}_{2}$ no interior das embalagens somado aos altos níveis de $\mathrm{CO}_{2}$ pode ter induzido os frutos a produzir energia pela respiração anaeróbia, resultando na produção de $\mathrm{CO}_{2}$, etanol e acetaldeído, muito mais evidente para o PP $30 \mu \mathrm{m}$. Neste sentido, constatou-se a presença de odor estranho nas embalagens $\mathrm{PP}$ a partir do $15 .^{\circ}$ dia de armazenamento refrigerado seguido por mais três dias sob condição ambiente, enquanto para o $\mathrm{PEBD}$, a presença de odor estranho foi observada a partir dos $30+3$ dias de armazenamento (dados não mostrados).

Além da presença de odor estranho nas embalagens, constatou-se que nos frutos acondicionados em PP houve alta incidência de manchas escuras na casca, o que pode ter sido promovida pelo acúmulo de altas concentraçóes de $\mathrm{CO}_{2}$ (Figura 2). Para os frutos testemunha, a incidência de manchas escuras aumentou a partir dos $30+3$ dias de armazenamento, enquanto os frutos acondicionados em PEBD permaneceram com baixa incidência de escurecimento durante todo o período. Sob tal aspecto, Ding et al. (2006) relataram que nêsperas 'Wuxing' armazenadas a $0{ }^{\circ} \mathrm{C}$ ou 6 ${ }^{\circ} \mathrm{C}$, em condiçôes de atmosfera modificada (PEBD $10 \mu \mathrm{m}$ ), tiveram atraso no aumento das atividades da polifenoloxidase e fenilalanina amonialiase, podendo estar relacionado à menor incidência de podridôes e de escurecimento. CAI et al. (2006b) correlacionaram o aumento do índice de escurecimento interno de nêsperas armazenadas a $0{ }^{\circ} \mathrm{C}$ ou a $20^{\circ} \mathrm{C}$ ao aumento da permeabilidade das membranas com a senescência, acompanhada da redução dos fenóis totais e aumento na atividade da polifenoloxidase.

A exposição de um fruto a níveis de $\mathrm{O}_{2}$ ou $\mathrm{CO}_{2}$ abaixo ou acima respectivamente, de seu limite de tolerância, resultará em estresse nos tecidos, manifestado por vários sintomas como, amadurecimento irregular, iniciação e/ou
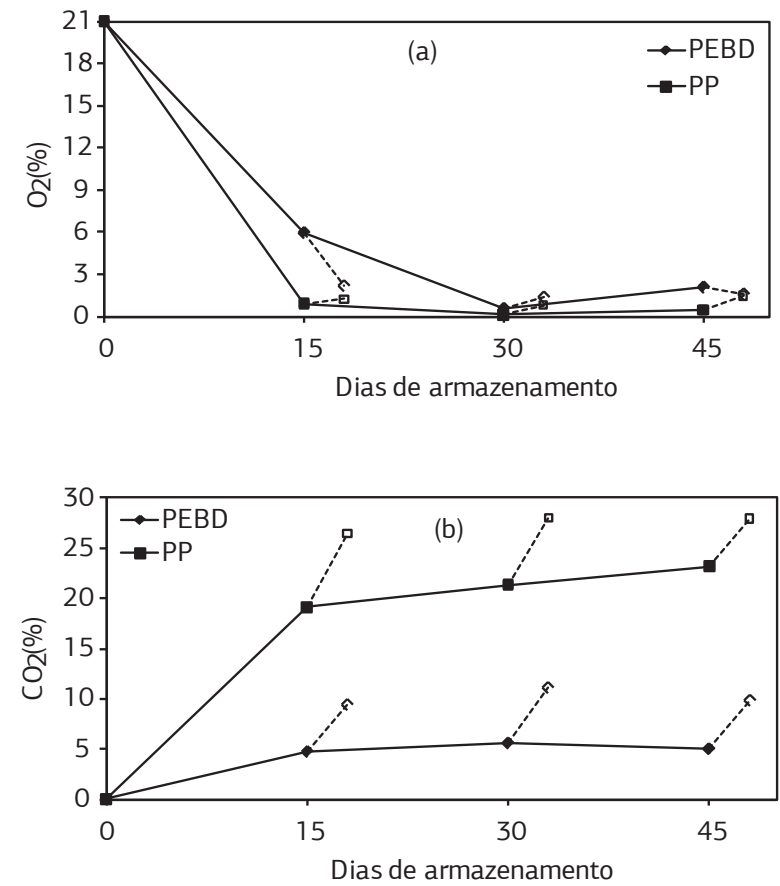

Figura 1. Níveis de $\mathrm{O}_{2}$ (a) e $\mathrm{CO}_{2}$ (b) no espaço-livre das embalagens de polietileno linear $50 \mu \mathrm{m}$ [PEBD $(\diamond)$ ] e polipropileno $30 \mu \mathrm{m}[\mathrm{PP}$ (घ)], durante o armazenamento refrigerado de nêsperas $\left(1{ }^{\circ} \mathrm{C} / 90 \%\right.$ UR), seguido pelo período de três dias sob condição ambiente (---- $\square$ ). Os pontos representam a média de quatro repetiçóes compostas por nove frutos por unidade experimental.

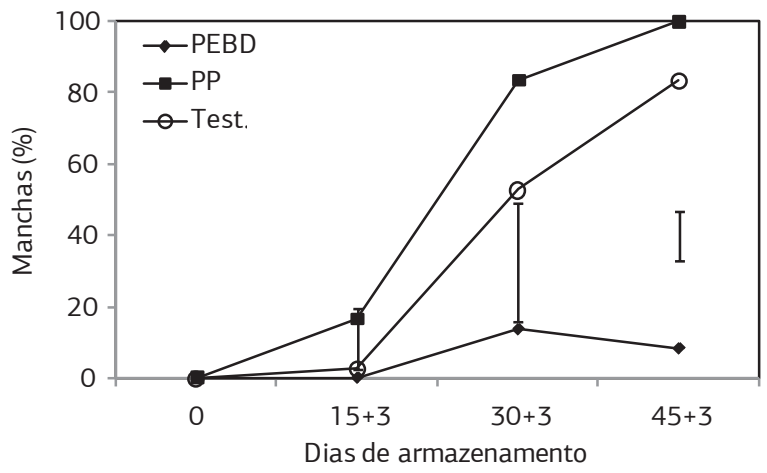

Figura 2. Incidência de manchas escuras na epiderme de nêsperas acondicionadas em polietileno linear $50 \mu \mathrm{m}$ [PEBD $(\downarrow)$ ] e polipropileno $30 \mu \mathrm{m}$ [PP ( $\mathbf{\square})$ ], durante o armazenamento refrigerado ( $\left({ }^{\circ} \mathrm{C} / 90 \%\right.$ UR), seguido pelo período de três dias sob condição ambiente. Testemunha [sem filme plástico (o)]. Linhas verticais indicam a diferença mínima significativa (DMS). Os pontos representam a média de quatro repetiçóes compostas por nove frutos por unidade experimental.

agravamento de certos distúrbios fisiológicos, desenvolvimento de sabor e odor desagradáveis e, aumento da suscetibilidade a doenças (KADER, 1986; LougheEd, 1987).

A incidência de podridóes permaneceu em baixos níveis nos frutos testemunha durante o período de armazenamento (5,50\%); nos frutos envoltos em PEBD e PP houve aos $45+3$ dias de armazenamento, $38,90 \%$ e $27,80 \%$ de incidência respectivamente. DING et al. (2002) consta- 
Tabela 1. Sólidos solúveis (SS), acidez titulável (AT), $\mathrm{pH}$, perda de massa e firmeza de nêsperas 'Fukuhara' acondicionadas em diferentes embalagens e armazenadas a $1{ }^{\circ} \mathrm{C} / 90 \%$ UR por até 45 dias seguido de mais três dias sob condição ambiente

\begin{tabular}{|c|c|c|c|c|c|c|}
\hline \multirow{2}{*}{ Data } & \multirow{2}{*}{ Tratamento } & SS & AT & Perda de massa & Firmeza & \multirow{2}{*}{$\mathrm{pH}$} \\
\hline & & & $\%$ & & $\mathbf{N}$ & \\
\hline & Dia 0 & 9,13 & 0,35 & 0 & 2,88 & 3,93 \\
\hline \multirow{3}{*}{$15+3^{x}$} & Testemunha & $8,90 \mathrm{a}$ & $0,23 \mathrm{a}$ & $7,52 \mathrm{a}$ & $3,38 \mathrm{a}$ & $4,34 \mathrm{a}$ \\
\hline & PEBD $50 \mu \mathrm{m}$ & $8,95 \mathrm{a}$ & $0,21 \mathrm{a}$ & $0,55 \mathrm{~b}$ & $2,69 \mathrm{a}$ & $4,38 \mathrm{a}$ \\
\hline & PP $30 \mu \mathrm{m}$ & $8,90 \mathrm{a}$ & $0,23 a$ & $0,64 b$ & $2,95 \mathrm{a}$ & $4,20 \mathrm{a}$ \\
\hline C.V. & & 8,01 & 23,25 & 20,40 & 14,80 & 3,49 \\
\hline \multirow{3}{*}{$30+3$} & Testemunha & $9,05 \mathrm{a}$ & $0,20 \mathrm{a}$ & $13,85 \mathrm{a}$ & $3,01 \mathrm{a}$ & $4,34 \mathrm{a}$ \\
\hline & PEBD $50 \mu \mathrm{m}$ & $8,15 \mathrm{a}$ & $0,20 \mathrm{a}$ & $0,57 \mathrm{~b}$ & $2,90 \mathrm{a}$ & $4,42 \mathrm{a}$ \\
\hline & PP $30 \mu \mathrm{m}$ & $8,40 \mathrm{a}$ & $0,18 a$ & $0,92 \mathrm{~b}$ & $3,15 \mathrm{a}$ & $4,40 \mathrm{a}$ \\
\hline C.V. & & 11,78 & 20,73 & 23,93 & 12,69 & 4,33 \\
\hline \multirow{3}{*}{$45+3$} & Testemunha & $10,15 a$ & $0,20 \mathrm{a}$ & $13,37 \mathrm{a}$ & $3,12 a b$ & $4,58 \mathrm{a}$ \\
\hline & PEBD $50 \mu \mathrm{m}$ & $8,20 a b$ & $0,20 \mathrm{a}$ & $0,72 \mathrm{~b}$ & $2,90 \mathrm{~b}$ & $4,67 \mathrm{a}$ \\
\hline & $\mathrm{PP} 30 \mu \mathrm{m}$ & $7,75 b$ & $0,16 \mathrm{a}$ & $1,34 \mathrm{~b}$ & $3,87 \mathrm{a}$ & $4,79 \mathrm{a}$ \\
\hline C.V. & & 11,71 & 20,78 & 12,93 & 13,93 & 3,78 \\
\hline
\end{tabular}

Médias seguidas de mesma letra, na coluna e em cada data de análise, nấo diferem significativamente entre si (Tukey, p $\leq 0,05)$. Período de armazenamento refrigerado $\left(1{ }^{\circ} \mathrm{C}\right)$, seguido de três dias sob condição ambiente $\left(25^{\circ} \mathrm{C}\right)$.

taram que nêsperas 'Mogi' acondicionadas em PEBD 50 $\mu \mathrm{m}$ tiveram $20 \%$ de incidência de podridóes após 60 dias de armazenamento, a $5{ }^{\circ} \mathrm{C}$. Quando armazenadas a 20 ${ }^{\circ} \mathrm{C}$, nesta mesma embalagem, houve $10 \%$ de incidência de podridóes após cinco dias, atingindo $100 \%$ após 21 dias. Os autores sugeriram que a alta incidência de podridóes foi devida à alta concentração de $\mathrm{CO}_{2}(12 \%)$ no interior de embalagens com baixa permeabilidade a gases. Relataram ainda que a utilização de atmosfera modificada não é adequada para o armazenamento de nêsperas sob condição ambiente. A utilização de atmosfera modificada pode resultar em algumas desvantagens, destacando-se a possível condensação de água no interior do filme, que pode favorecer o crescimento de fungos e aumentar os problemas devido a doenças (KADER et al., 1989, SARANTópoulos et al., 1996).

$\mathrm{Na}$ tabela 1 nota-se que as duas embalagens avaliadas foram eficientes em limitar a perda de massa de nêsperas durante o período de armazenamento dos frutos. A redução da perda de massa está diretamente relacionada à taxa de transmissão de vapor d'água da embalagem. Quanto menor a taxa de transmissão, menor o déficit de pressão de vapor d'água e maior a umidade relativa no interior da embalagem, reduzindo a taxa de transpiração das frutas. De forma semelhante, Ding et al. (2002) observaram que nas nêsperas acondicionadas em PEBD ocorreu cerca de 1,5\% de perda de massa após 60 dias de armazenamento a $5{ }^{\circ} \mathrm{C}$.

As embalagens utilizadas não alteraram a acidez titulável, o pH e a firmeza das nêsperas durante o período de armazenamento; somente os teores de sólidos solúveis estavam menores para os frutos acondicionados em PP, após $45+3$ dias de armazenamento. Independentemente da embalagem utilizada, não se constataram alteraçóes destes atributos durante o período de armazenamento. Sob tal aspecto, Ding et al. (1998) observaram significativa reduçáo no conteúdo de ácido málico em nêsperas armazenadas a $5{ }^{\circ} \mathrm{C}$. Posteriormente, Ding et al. (2002) relataram que a redução da concentração de ácidos orgânicos foi mais lenta para os frutos acondicionados em PEBD. Segundo CaI et al. (2006b), o aumento da firmeza é característico da nêspera durante o amadurecimento e a senescência, e afirmam que está associado ao aumento da lignificação, que ocorre tanto em condiçóes ambientes quanto em armazenamento refrigerado.

Desta forma, trabalhos deverão ser desenvolvidos visando à melhoria do filme a ser utilizado (tipo, espessura e permeabilidade) a fim de se obter uma atmosfera adequada ao redor dos frutos para o aumento do período de conservação dos mesmos, evitando o desenvolvimento de podridōes.

\section{CONCLUSÃO}

A utilização de filme PEBD $(50 \mu \mathrm{m})$ ou PP $(30 \mu \mathrm{m})$, associada ao armazenamento refrigerado a $1{ }^{\circ} \mathrm{C} / 90 \% \mathrm{UR}$, para a conservação pós-colheita da nêspera 'Fukuhara', não é indicada para o aumento do período de conservação dos frutos, pois deprecia sua qualidade devido a condiçóes de anaerobiose e elevada incidência de podridôes.

\section{AGRADECIMENTOS}

Ao Conselho Nacional de Desenvolvimento Científico e Tecnológico - CNPq, pelo auxílio financeiro ao desenvolvimento do projeto. 


\section{REFERÊNCIAS}

BARBOSA, W.; POMMER, C.V.; RIBEIRO, M.D.; VEIGA, R.F.A.; COSTA, A.A. Distribuição geográfica e diversidade varietal de frutíferas e nozes de clima temperado no Estado de São Paulo. Revista Brasileira de Fruticultura, v.25, p.341-344, 2003.

BRACKMANN, A.; SAQUET, A.A.; CERETTA, M. Qualidade de nêspera (Eriobotrya japonica, Lindl.) armazenada em diferentes temperaturas e concentraçōes de $\mathrm{CO}_{2}$ e $\mathrm{O}_{2}$. Revista Brasileira de Agrociência, v.2, p.183-186, 1996.

CAI, C; XU, C.; SHAN, L.; LI, X.; ZHOU, C.; ZHANG, W.; FERGUSON, I.; CHEN, K. Low temperature conditioning reduces postharvest chilling injury in loquat fruit. Postharvest Biology and Technology, v. 41, p. 252-259, 2006 a.

CAI, C; CHEN, K.; XU, W.; ZHANG, W.; LI, X.; FERGUSON, I. Effect of 1-MCP on postharvest quality of loquat fruit. Postharvest Biology and Technology, v. 40, p. 165-162, 2006b.

CIA, P.; BENATO, E.A. Controle Alternativo com Atmosferas Modificada e Controlada. In: OLIVEIRA, S.M.A.; TERAO, D.; DANTAS, S.A.F.; TAVARES, S.C.C.H. (Org.). Patologia PósColheita: Frutas, Olerícolas e Ornamentais Tropicais. 1 ed. Brasília: Embrapa Informação Tecnológica, 2006, p. 247-264.

DING, Z.; TIAN, S.; WANG, Y.; LI, O.; CHAN, Z.; HAN, J. $\mathrm{XU}, \mathrm{Y}$. Physiological response of loquat fruit to different storage conditions and its storability. Postharvest Biology and Technology, v. 41, p.143-150, 2006.

DING, C.K.; CHACHIN, K.; UEDA, Y.; IMAHORI, Y.; WANG, C.Y. Modified atmosphere packaging maintains postharvest quality of loquat fruit. Postharvest Biology and Technology, v.24, p.341$348,2002$.

DING, C.K.; CHACHIN, K.; HAMAUZU, Y.; UEDA, Y.; IMAHORI, Y. Effects of storage temperatures on physiology and quality of loquat fruit. Postharvest Biology and Technology, v.14, p.309-315, 1998.

FARIA, A.F.; HASEGAWA, P.N.; CHAGAS, E.A.; PIO, R.; PURGATTO, E.; MERCADANTE, A.Z. Cultivar influence on carotenoid composition of loquats from Brazil. Journal of Food Composition and Analysis, v.22, p.196-203, 2009.

FERRERES, F.; GOMES, D.; VALENTÁO, P.; GONÇALVES, R.; PIO, R.; CHAGAS, E.A.; SEABRA, R.M.; ANDRADE, P.B. Improved loquat (Eriobotrya japonica Lindl.) cultivars: Variation of phenolics and antioxidative potential. Food Chemistry, v.114, p.1019-1027, 2009.

KADER, A.A. Biochemical and physiological basis for effects of controlled and modified atmosphere on fruits and vegetables. Food Technology, v.40, p.99-103, 1986.

KADER, A.A.; ZAGORY, D.; KERBEL, E.L. Modified atmosphere packaging of fruits and vegetables. Critical Reviews in Food Science and Nutrition, v. 28, p.1-30, 1989.

LIN, S.; SHARPE, R.H.; JANICK, J. Loquat: botany and horticulture. Horticultural Reviews, v. 23, p. 234-276, 1999.

LOUGHEED, E.C. Interactions of oxigen, carbon dioxide, temperature, and ethylene that may induce injuries in vegetables. HortScience, v.22, p.791-794, 1987.

MATHOOKO, F.M. Regulation of respiration metabolism in fruits and vegetables by carbon dioxide. Postharvest Biology and Technology, v.9, p.247-64, 1996.

MELO, A.A.M.; LIMA, L.C.O. Influência de três diferentes embalagens de PVC na vida pós-colheita de nêspera. Ciência e Agrotecnologia, v.27, p.1330-1339, 2003.

PIO, R.; CAMPO DALL'ORTO, F.A.; CHAGAS, E.A.; BARBOSA, W. Aspectos técnicos do cultivo de nêsperas. Piracicaba: ESALQ/ USP, 2008. 30p. (Série Produtor Rural, 39)

PIO, R.; CAMPO DALL'ORTO, F.A.; BARBOSA, W.; CHAGAS, E.A.; OJIMA, M.; CIA, P. Produção de cultivares de nespereira na região leste paulista. Pesquisa Agropecuária Brasileira, v.42, p.10531056, 2007.

SARANTÓPOULOS, C.I.G.L.; ALVES, R.M.V.; OLIVEIRA, L.M.; GOMES, T.C. Embalagens com atmosfera modificada. Campinas: Instituto de Tecnologia de Alimentos, 1996. 114p. 